\title{
Cluster lag synchronization of complex networks with nonidentical dynamical nodes via Adaptive control Shuguo Wang ${ }^{\mathrm{a}}$, Haitao $\mathrm{Cao}^{\mathrm{b}}$
}

\author{
Department of Mathematics and Physics, Changzhou Campus, Hohai University, Changzhou \\ 213022, PR China \\ aWsg97@163.com b5882450@qq.com
}

Keywords: Cluster lag synchronization, Complex networks, Adaptive control.

\begin{abstract}
In this paper, synchronization analysis is investigated for an complex networks with time delay. It is assumed the nodes in the network to be nonidentical. In order to achieve cluster synchronization, this paper uses an adaptive controller on each node, by utilizing the Lyapunov stability theory, a new and useful synchronization criteria are obtained, which guarantee the complex networks under control could realize the cluster lag synchronization. Finally, numerical simulation results are also presented to verify the effectiveness of the proposed synchronization scheme.
\end{abstract}

\section{Introduction}

As it is well known, synchronization and control in complex networks have been known for a rather long time, and many kinds of synchronization have been introduced[1-8], such as complete synchronization, lag synchronization, projective synchronization, impulsive synchronization , cluster synchronization, partial synchronization, mixed synchronization, and so on.

Cluster synchronization in community network with nonidentical nodes is studied and several sufficient conditions for synchronization are obtained analytically. In [9], complex networks with community structure and nonidentical nodes are investigated. The node dynamics of different communities in the same community network are assumed to be nonidentical. Based on Lyapunov stability theory, sufficient conditions for achieving cluster projective synchronization are derived through designing proper controllers. In [10], $H 1$ cluster synchronization and state estimation for complex dynamical networks with mixed time delays is investigated. A novel concept of $H 1$ cluster synchronization is proposed to quantify against the exogenous disturbance of the complex networks. In [11], cluster synchronization in nonlinearly coupled delayed networks of non-identical dynamic systems is investigated. In [12], complex networks with community structure and nonidentical nodes are investigated. The cluster mixed synchronization of these networks is studied by using some linear pinning control schemes. In [13], cluster synchronization under the influence of advection is considered. In [14], Liu et al. investigated cluster synchronization for networks with cooperative and competitive weight couplings. In [15], cluster synchronization is studied in adaptive complex dynamical networks with nonidentical nodes and a novel adaptive strategy is proposed to realize global cluster synchronization.

Motivated by the above discussions, this paper investigates cluster lag synchronization in the time delay coupled complex dynamical networks. For achieving the synchronization, basing on the structure of the networks and Lyapunov stability theory, this paper uses an adaptive controller on each node, some sufficient criteria are derived to ensure cluster lag synchronization.

The paper is organized as follows: The network model is introduced followed by some definitions, lemmas and hypotheses in section 2. The cluster lag synchronization of the complex coupled networks is discussed in section 3. Simulations are obtained in section 4. Finally, in section five the various conclusions are discussed.

\section{Model and preliminaries}

The network with delay coupling can be described by 


$$
\dot{x}_{i}(t)=f_{\phi_{i}}\left(t, x_{i}(t)\right)+c \sum_{j=1}^{N} b_{i j} \Gamma x_{j}(t-\tau), \quad i=1,2, \ldots, N
$$

where $x_{i}(t)=\left(x_{i 1}(t), x_{i 2}(t), \cdots, x_{i n}(t)\right)^{T} \in R^{n}$ is the state vector of node $i, c>0$ is the coupling strength. $f_{\phi_{i}}: R^{n} \rightarrow R^{n}$ describes the local dynamics of nodes in the $\phi_{i}$ th community. For any pair of nodes $i$ and $j$, if $\phi_{i} \neq \phi_{j}$, i.e., nodes $i$ and $j$ belong to different communities, then $f_{\phi_{i}} \neq f_{\phi_{j}}$. $\Gamma \in R^{n \times n}$ is inner-coupling matrices, $\tau>0$ is the time delay, $B=\left(b_{i j}\right)_{N \times N}$ are the weight configuration matrices. If there is a connection from node $i$ to node $j(j \neq i)$ then $b_{i j}>0$, otherwise, $b_{i j}=b_{j i}=0$, and the diagonal elements of matrix $B$ are defined as

$$
b_{i i}=-\sum_{j=1, j \neq i}^{N} b_{i j}, \quad i=1,2, \cdots, N
$$

When the control inputs $v_{i}(t)$ and $u_{i}(t) \in R^{n}(i=1,2, \cdots, N)$ are introduced, the controlled dynamical network with respect to network (1) can be written as

$$
\left\{\begin{array}{l}
\dot{x}_{i}(t)=f_{\phi_{i}}\left(t, x_{i}(t)\right)+c \sum_{j=1}^{N} b_{i j} \Gamma x_{j}(t-\tau)+u_{i}(t), \quad \phi_{i}(t) \in \bar{J}_{\phi_{i}} \\
\dot{x}_{i}(t)=f_{\phi_{i}}\left(t, x_{i}(t)\right)+c \sum_{j=1}^{N} b_{i j} \Gamma x_{j}(t-\tau)+v_{i}(t), \quad \phi_{i}(t) \in J_{\phi_{i}}-\bar{J}_{\phi_{i}}
\end{array}, i=1,2, \ldots, N\right.
$$

where $J_{\phi_{i}}$ denotes all the nodes in the $\phi_{i}$ th community and $\bar{J}_{\phi_{i}}$ represents the nodes in the $\phi_{i}$ th community which have direct links with the nodes in other communities.

Definition1. Let $\left\{C_{1}, C_{2}, \cdots, C_{m}\right\}$ denote $m(2 \leq m \leq N)$ communities of the networks and $\bigcup_{i=1}^{m} C_{i}=\{1,2, \cdots N\}$. Assume that $s(t) \in R^{n}$ is any smooth dynamics. The error variables as $e_{i}(t)=x_{i}(t)-s\left(t-\tau_{\phi_{i}}\right)$ for $i=1,2, \ldots, N$, the controlled complex community network (1) is said to achieve cluster lag synchronization $(C L S)$, if $\lim _{t \rightarrow \infty}\left\|x_{i}(t)-s\left(t-\tau_{\phi_{i}}\right)\right\|=0, i=1,2, \ldots, N$ for any initial conditions. where $\tau_{\phi_{i}}>0$ is the time delay

Lemma1. For any two vectors $x$ and $y$, a matrix $Q>0$ with compatible dimensions, one has: $2 x^{T} y \leq x^{T} Q x+y^{T} Q^{-1} y$.

Assumption 1 [16] (Global Lipschitz Condition). Suppose that there exist nonnegative constants $\gamma$, for all $\forall t \in R_{+}$, such that for any time-varying vectors $x(t), y(t) \in R^{n}$,

$\left\|f_{\phi_{i}}(x)-f_{\phi_{i}}(y)\right\| \leq \gamma\|x-y\|$,

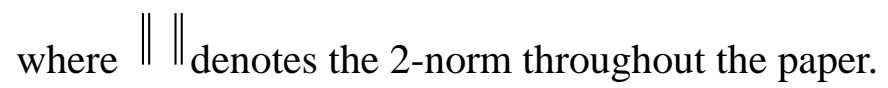

\section{Main results}

According to system (1) and Definition1, the error dynamical system can be derived as

$$
\left\{\begin{array}{c}
\dot{e}_{i}(t)=f_{\phi_{i}}\left(t, x_{i}(t)\right)-f_{\phi_{i}}\left(t, s\left(t-\tau_{\phi_{i}}\right)\right)+c \sum_{j=1}^{N} b_{i j} \Gamma e_{j}(t-\tau) \\
+c \sum_{i=1}^{N} b_{i j} \Gamma s\left(t-\tau_{\phi_{i}}\right)+u_{i}(t) \quad \phi_{i}(t) \in \bar{J}_{\phi_{i}}, i=1,2, \ldots, N . \\
\dot{e}_{i}(t)=f_{\phi_{i}}\left(t, x_{i}(t)\right)-f_{\phi_{i}}\left(t, s\left(t-\tau_{\phi_{i}}\right)\right)+c \sum_{j=1}^{N} b_{i j} \Gamma e_{j}(t-\tau) \\
\phi_{i}(t) \in J_{\phi_{i}}-\bar{J}_{\phi_{i}}
\end{array}\right.
$$


According to the diffusive coupling condition (2) of the matrix $A, B$ we have

$$
c \sum_{i=1}^{N} b_{i j} \Gamma s\left(t-\tau_{\phi_{i}}\right)=0, i \in J_{\phi_{i}}-\bar{J}_{\phi_{i}}
$$

On the basis of this property, for achieving cluster synchronization, we design the controllers as follows:

$$
\left\{\begin{array}{l}
u_{i}(t)=-c \sum_{i=1}^{N} b_{i j} \Gamma s\left(t-\tau_{\phi_{i}}\right)-d_{i} e_{i}(t), \quad i \in \bar{J}_{\phi_{i}} \\
v_{i}(t)=-d_{i} e_{i}(t), \quad i \in J_{\phi_{i}}-\bar{J}_{\phi_{i}}
\end{array}\right.
$$

where $\dot{d}_{i}=k_{i} e_{i}^{T}(t) e_{i}(t), d_{i}$ are the feedback strength and $k_{i}$ are arbitrary positive constants.

Theorem 1. Suppose Assumptions 1 hold. Consider the network (1) via control law (6). If the following conditions hold:

$$
\lambda_{\max }\left(P P^{T}\right)<\frac{2}{c}(d-\gamma)-1
$$

Then, the system (3) is cluster lag synchronization. Where $d$ is a sufficiently large positive constant to be determined.

Proof. Construct the following Lyapunov functional

$$
V(t)=\frac{1}{2} \sum_{i=1}^{N} e_{i}^{T}(t) e_{i}(t)+\frac{c}{2} \int_{t-\tau}^{t} \sum_{i=1}^{N} e_{i}^{T}(\xi) e_{i}(\xi) d \xi+\frac{1}{2} \sum_{i=1}^{N} \frac{\left(d_{i}-d\right)^{2}}{k_{i}}
$$

Calculating the derivative of $V(t)$, we have

$$
\begin{aligned}
\dot{V}(t)= & \sum_{i=1}^{N} e_{i}^{T}(t)\left[f_{\phi_{i}}\left(t, x_{i}(t)\right)-f_{\phi_{i}}\left(t, s\left(t-\tau_{\phi_{i}}\right)\right)+c \sum_{j=1}^{N} b_{i j} \Gamma e_{j}(t-\tau)-d_{i} e_{i}(t)\right]+\frac{c}{2} \sum_{i=1}^{N} e_{i}^{T}(t) e_{i}(t) \\
& -\frac{C}{2} \sum_{i=1}^{N} e_{i}^{T}(t-\tau) e_{i}(t-\tau)+\sum_{i=1}^{N}\left(d_{i}-d\right) e_{i}^{T}(t) e_{i}(t) \\
\leq & \sum_{i=1}^{N} e_{i}^{T}(t)\left[f_{\phi_{i}}\left(t, x_{i}(t)\right)-f_{\phi_{i}}\left(t, s\left(t-\tau_{\phi_{i}}\right)\right)\right]+c \sum_{i=1}^{N} \sum_{j=1}^{N} b_{i j} e_{i}^{T}(t) \Gamma e_{j}\left(t-\tau_{1}\right)+\frac{C}{2} \sum_{i=1}^{N} e_{i}^{T}(t) e_{i}(t) \\
& -\frac{c}{2} \sum_{i=1}^{N} e_{i}^{T}(t-\tau) e_{i}(t-\tau)-\sum_{i=1}^{N} d e_{i}^{T}(t) e_{i}(t)
\end{aligned}
$$

By the Assumption1, we have

$$
\begin{aligned}
\dot{V}(t) & \leq \sum_{i=1}^{N} e_{i}^{T}(t) \gamma e_{i}(t)+c e^{T}(t)(B \otimes \Gamma) e(t-\tau)+\frac{c}{2} e^{T}(t) e(t)-\frac{c}{2} e^{T}(t-\tau) e(t-\tau) \\
& -\sum_{i=1}^{N} d e_{i}^{T}(t) e_{i}(t)
\end{aligned}
$$

Let $e(t)=\left(e_{1}^{T}(t), e_{2}^{T}(t), \ldots, e_{N}^{T}(t)\right)^{T} \in R^{n N}, \quad P=(B \otimes \Gamma)$, where $\otimes$ represents the Kronecker product. Then

$$
\dot{V}(t) \leq c e^{T}(t) P e(t-\tau)+\frac{c}{2} e^{T}(t) e(t)-\frac{c}{2} e^{T}(t-\tau) e(t-\tau)+\gamma e^{T}(t) e(t)-d e^{T}(t) e(t)
$$

By the Lemma1, we have

$$
\begin{aligned}
\dot{V}(t) & \leq \frac{c}{2} e^{T}(t) P P^{T} e(t)+\frac{c}{2} e^{T}(t) e(t)+\gamma e^{T}(t) e(t)-d e^{T}(t) e(t) \\
& \leq\left(\gamma+\frac{c}{2} \lambda_{\max }\left(P P^{T}\right)+\frac{c}{2}-d\right) e^{T}(t) e(t)
\end{aligned}
$$

Therefore, if we have $\lambda_{\max }\left(P P^{T}\right)<\frac{2}{c}(d-\gamma)-1$ then $\dot{V}(t) \leq 0$.Cluster lag synchronization in the network (3) is achieved under the controllers (6), this completes the proof. 


\section{Illustrative examples}

In this section, the nodes dynamics are the following well-known modified Chua's circuit [17] with different system parameters considering the network (3). $x_{i}(t)=\left(x_{1 i}(t), x_{2 i}(t), x_{3 i}(t)\right)^{T}$, $f_{1}\left(t, x_{i}(t)\right)=D_{1} x_{i}(t)+h_{1}\left(x_{i}(t)\right) \quad, f_{2}\left(t, x_{i}(t)\right)=D_{2} x_{i}(t)+h_{2}\left(x_{i}(t)\right), \quad f_{3}\left(t, x_{i}(t)\right)=D_{3} x_{i}(t)+h_{3}\left(x_{i}(t)\right)$, $h_{1}\left(x_{i}\right)=h_{2}\left(x_{i}\right)=h_{3}\left(x_{i}\right)=\left(-\frac{20}{7} x_{i 1}^{3}, 0,0\right)^{T}, \Gamma=\operatorname{Diag}\{1,1,1\} \quad$ and $\quad \tau=0.6, \quad c=2, d=15$, $D_{1}=\left[\begin{array}{ccc}-10 / 7 & 10 & 0 \\ 1 & -1 & 1 \\ 0 & -12 & 0\end{array}\right] \quad, \quad D_{2}=\left[\begin{array}{ccc}-10 / 7 & 10 & 0 \\ 1 & -1 & 1 \\ 0 & -13 & 0\end{array}\right] \quad, \quad D_{3}=\left[\begin{array}{ccc}-10 / 7 & 10 & 0 \\ 1 & -1 & 1 \\ 0 & -14 & 0\end{array}\right]$ $B=\left[\begin{array}{cccccc}-1 & 1 & 0 & -1 & 0 & 1 \\ 0 & -2 & 0 & 1 & 0 & 1 \\ 1 & 0 & -3 & 1 & 0 & 1 \\ 0 & 1 & 0 & -2 & 1 & 0 \\ 1 & 0 & 1 & 0 & -3 & 1 \\ 0 & 1 & 0 & 1 & 0 & -2\end{array}\right]$.

The following quantities are utilized to measure the process of cluster lag synchronization

$$
E(t)=\sum_{i=1}^{12}\left\|x_{i}(t)-s\left(t-\tau_{\phi_{i}}\right)\right\|, \phi_{i}=1,2,3
$$

where $E(t)$ is the error of cluster lag synchronization for this controlled network (2), cluster lag synchronization is achieved if the synchronization error $E(t)$ converges to zero as $t \rightarrow \infty$. Let $\tau_{1}=0.5, \tau_{2}=1, \tau_{3}=1.2$. Simulation results are given in Fig.1-2. Fig.1 shows the time evolution of the total synchronization errors $E(t)$. Fig.2 shows orbits of the adaptive feedback strength. The numerical results show that Theorem 1 is effective.

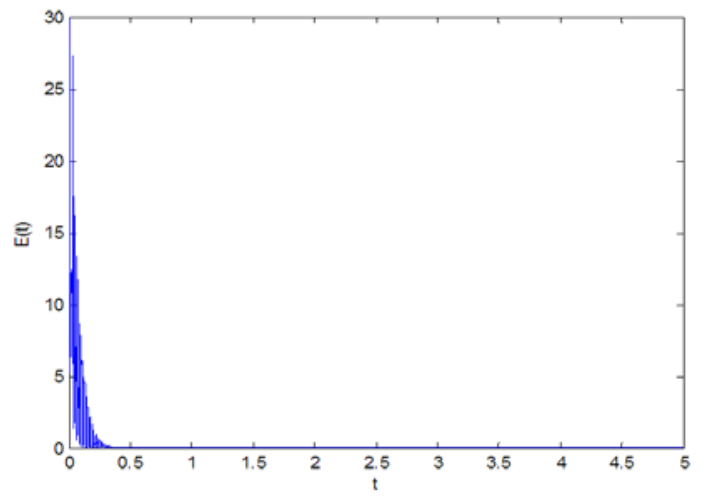

Fig.1. Time evolution of the synchronization errors $E(t)$.

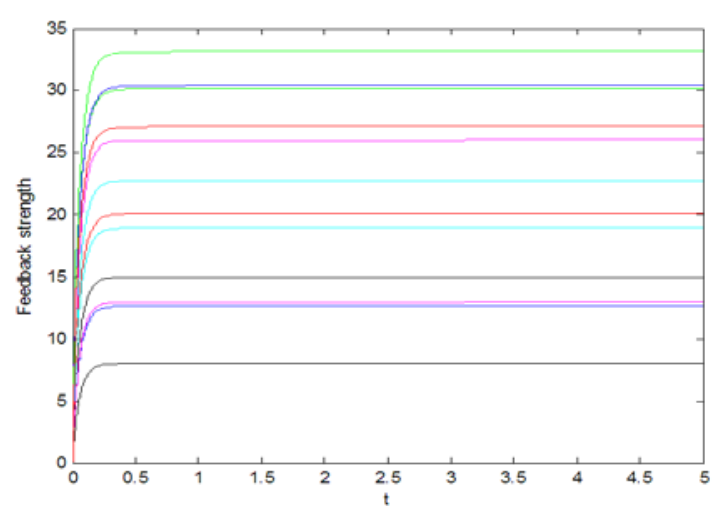

Fig.2. Orbits of the adaptive feedback strength $d_{i}$.

\section{Conclusions}

The cluster lag synchronization in community networks has been studied in this paper. According to Lyapunov stability theory, some simple and useful criteria are derived to ensure cluster lag synchronization. Particularly, the weight configuration matrix is not assumed to be symmetric and irreducible. Finally, the developed techniques are applied three complex community networks. 
Numerical simulations are provided to verify the correctness and effectiveness of the derived theoretical results.

\section{Acknowledgments}

Research is partially supported by the Fundamental Research Funds for the Central Universities, China (no. 2013B10014, 2013B10114).

\section{References}

[1] Lu J, Cao J, Adaptive feedback synchronization of uncertain dynamical networks with delayed coupling. Nonlinear Dynam, 53, 107-115, 2008.

[2] Zheng S, Dong G G, Bi Q S. Impulsive synchronization of complex networks with non-delayed and delayed coupling. Phys Lett A, 373:4255-9, 2009.

[3] Wang Y, Wang Z, Liang J, Global synchronization for delayed complex networks with randomly occurring nonlinearities and multiple stochastic disturbances, J. Phys. A: Math. Theor, 42, 135101, 2009.

[4] Lu W L, Liu B, Chen T P, Cluster synchronization in networks of coupled nonidentical dynamical systems. Chaos, 20:013120, 2010.

[5] Wang S, Yao H, Bian Q, Synchronization in complex networks with time-varying delays dynamical nodes via pinning control, Math. Probl. Eng. 654742, 2012.

[6] Hu M F, Xu Z Y, Yang Y Q, Projective cluster synchronization in drive-response dynamical networks. Physica A, 387:3759-68, 2008.

[7] Wang J W, Ma Q H, Zeng L, Abd-Elouahab MS. Mixed outer synchronization of coupled complex networks with time-varying coupling delay. Chaos , 21:013121, 2011.

[8] Guirey E, Bees M, Martin A, Srokosz M. Persistence of cluster synchronization under the influence of advection. Phys Rev E, 81:051902, 2010.

[9] $\mathrm{Wu} \mathrm{Z} \mathrm{Y,} \mathrm{Fu} \mathrm{X} \mathrm{C,} \mathrm{Cluster} \mathrm{projective} \mathrm{synchronization} \mathrm{between} \mathrm{community} \mathrm{networks} \mathrm{with}$ nonidentical nodes, Physica A, 391, 6190-6198, 2012.

[10]Li H J, H1 cluster synchronization and state estimation for complex dynamical networks with mixed time delays, Applied Mathematical Modelling, 37, 12-13, 7223-7244, 2013.

[11]Wang Y L, Cao J D ,Cluster synchronization in nonlinearly coupled delayed networks of non-identical dynamic systems, Nonlinear Analysis: Real World Applications, 14, 842-851, 2013.

[13]Guirey E, Bees M, Martin A, Srokosz M. Persistence of cluster synchronization under the influence of advection. Phys Rev E , 81:051902, 2010.

[14] Liu X, Chen T, Cluster synchronization in directed networks via intermittent pinning control, IEEE Trans. Neural Netw. 22, 1009-1020, 2011.

[15]Wu X, Lu H, Cluster synchronization in the adaptive complex dynamical networks via a novel approach, Phys. Lett. A. 375, 1559-1565, 2011.

[16]Liu H, Chen J, Lu J A and Cao M, Generalized synchronization in complex dynamical networks via adaptive couplings, Physica A, Volume 389, Issue 8, pp. 1759-1770, 2010.

[17]Wang J W, Ma Q H, Zeng L, Abd-Elouahab MS. Mixed outer synchronization of coupled complex networks with time-varying coupling delay, Chaos, 21, 013121, 2011. 